\title{
Geoparques mundiales de la UNESCO y su importancia en el desarrollo sostenible de las comunidades. Estudio de caso: "Geoparque Imbabura"
}

\section{UNESCO's Global Geoparks and its importance on communities' sustainable development. Case study: "Geopark Imbabura"}

Sonia Lorena Arellano Guerrón ${ }^{1}$, Dayané Mabel Arroyo Mera ${ }^{1}$, Edwin Agustín Carrión Albuja ${ }^{1}$, Carlos Ernesto Merizalde Leiton²

凶Autorde correspondencia: slarellano@pucesi.edu.ec,dmarroyo@pucesi,edu.ec, eacarrion@pucesi.edu.ec, cmerizalde@imbabura.gob.ec

${ }^{1}$ Pontificia Universidad Católica del Ecuador, sede Ibarra. Av. Aurelio Espinosa Pólit y Av. Jorge Guzmán Rueda. Cdla. La Victoria. Ibarra. 100105. Ecuador

${ }^{2}$ Gobierno Provincial de Imbabura. Antonio José de Sucre y García Moreno. Ibarra. 100105. Ecuador.

Artículo original de la ponencia presentada en el IV CONGRETUR - "El impacto del turismo en el desarrollo del Ecuador", Universidad Central del Ecuador, 21 al 24 de noviembre de 2018

\begin{abstract}
Resumen
La provincia de Imbabura, en el año 2015 presentó su candidatura para ser reconocida como Geoparque Mundial de la UNESCO; luego de una primera valoración y después de haber acogido las sugerencias hechas por la comisión evaluadora, se presentó el dossier definitivo en febrero del 2018. El proyecto Geoparque Imbabura reúne nueve complejos volcánicos, el nombre se asocia con las raíces históricas, culturales y lingüísticas de la provincia donde el idioma kichwa y las costumbres tradicionales indígenas siguen siendo valores de uso cotidiano. El proyecto es un proceso participativo que busca consolidar su identidad cultural, fundamentada en el reconocimiento, respeto y conservación de su patrimonio geológico, arqueológico, cultural, con las formas de vida, cosmovisiones, costumbres y tradiciones de sus habitantes, para la implementación de un modelo de desarrollo territorial sustentable, que lleve a su población a una mejor calidad de vida.
\end{abstract}

Palabras clave: Geoturismo, Desarrollo Sostenible, Conservación del Patrimonio, Geositios.

\begin{abstract}
On 2015, Imbabura province submitted its candidacy to be recognized as UNESCO World Geopark; after a first assessment and after having accepted the suggestions made by the evaluation committee; the final Dossier was presented in February 2018. The Imbabura Geopark Project brings together nine volcanic complexes; the name is associated with the historical, cultural and linguistic roots of the province where the Kichwa language and traditional indigenous customs are still considered as everyday values. The project is a participatory process that seeks to consolidate its cultural identity based on the recognition, respect and conservation of its geological, archaeological, culture, with the different ways of life, Cosmo visions, customs, and traditions of such people, for the implementation of a model of sustainable territorial development that leads its population to a better quality of life.
\end{abstract}

Keywords: Geotourism; Sustainable development; Heritage Conservation; Geosites. 


\section{Introducción}

Un geoparque (geopark) es un territorio que presenta un patrimonio geológico notable que es el eje fundamental de una estrategia de desarrollo territorial sostenible basado en la educación y el turismo (Carcavilla Urquí \& Cortés, 2018); desde el 17 de noviembre de 2015, la Conferencia General de la UNESCO con sus 193 Estados Miembros, tomó la decisión de crear oficialmente esta nueva etiqueta "Geoparques Mundiales de la UNESCO" para valorar y reconocer la importancia y diversidad del patrimonio geológico en los territorios, en su evolución de 4.600 millones de años del planeta Tierra. UNESCO (2018). Actualmente existen 140 geoparques mundiales reconocidos por UNESCO. En las Américas, existen cuatro: Araripe, en Brasil; Grutas del Palacio, en Uruguay; Mixteca Alta y Comarca Minera, en México.

Según datos del Consejo Nacional de Límites (CONALI), el territorio del aspirante Geoparque Imbabura, citado en el Plan de Desarrollo y Ordenamiento Territorial de la Provincia de Imbabura, tiene una superficie de 4.619,03 km2 o su equivalente, 461.900 hectáreas, aproximadamente. Imbabura, según las proyecciones del Instituto Ecuatoriano de Estadística y Censos con base en el Censo del 2010, tiene una población al 2015 , de 428.355 habitantes. Está organizada en 6 cantones: Ibarra, capital provincial, fundada en 1606; Otavalo, Cotacachi, Antonio Ante, Urcuquí y Pimampiro. Su organización política, incluye 36 parroquias rurales. Las condiciones orográficas de la provincia, determinan una diversidad climática y eco sistémica, que constituyen la base natural para el desarrollo productivo y turístico. Los tipos de clima presentes son: cálido seco en el Valle del Chota, pasando por el templado en las cabeceras cantonales, a frío de alta montaña en los volcanes Imbabura y Cotacachi. Los sectores de Íntag y Lita se caracterizan por tener un clima cálido húmedo.

El Proyecto Geoparque Imbabura (PGI) es un proceso participativo que busca: consolidar una identidad cultural, fundamentada en el reconocimiento, respeto y conservación de su patrimonio geológico, con las formas de vida, cosmovisión, costumbres y tradiciones de sus pueblos; determinar las potencialidades geológicas para el desarrollo del geoturismo, la educación y la conservación del patrimonio en la provincia, capitalizando los aspectos claves de las fortalezas territoriales como ubica- ción geográfica, aspectos culturales, naturales; todo ello con el diseño e implementación de un modelo de desarrollo territorial sustentable y diversificación de la oferta turística que lleve a su población a una mejor calidad de vida.

Sin embargo, hasta antes del 2015, año en el que se empieza a desarrollar interés por gestionar este macroproyecto, no se habían planteado procesos integrales a largo plazo que permitieran afianzar el crecimiento económico con criterios de sostenibilidad y que generaran un mejoramiento de la calidad de vida de la población, especialmente en sectores periurbanos y rurales.

El PGI tiene como objetivo general, alcanzar la denominación para la provincia de Imbabura como «Geoparque Mundial de la UNESCO» como instrumento para el fortalecimiento de la identidad y desarrollo local y nacional. Para el logro de este objetivo, se han planteado algunos objetivos secundarios entre los cuales están: organizar, fundamentar y socializar el proyecto; formular y ejecutar un plan que abarque y desarrolle como ejes esenciales, desde la perspectiva geológica, los componentes de educación, turismo y conservación; diseñar e implementar un modelo de gestión para que dé sostenibilidad al proyecto, que promueva el trabajo colaborativo entre los actores locales: Comité de Gestión, INIGEMM, UC, YACHAY TECH, IG EPN, Comisión Provincial de Turismo, academia local, gobiernos autónomos descentralizados (GADs), actores y agentes privados, comunidad; formular el documento dossier Proyecto Geoparque Imbabura para su postulación ante la UNESCO.

Por lo tanto, se hace necesario emprender acciones para que la comunidad sea capaz de concienciar, caracterizar, valorar, defender y conservar su propio entorno. El PGI invita a desarrollar capacidades y buenas prácticas en el aprovechamiento racional de sus recursos. A través de la denominación de Imbabura como GEOPARQUE MUNDIAL DE LA UNESCO, el turismo podrá poner en valor el rico patrimonio geológico existente; a partir del conocimiento del patrimonio geológico, se deberá cambiar el rumbo de la interpretación del patrimonio con fines de guianza turística, tomando elementos (geología) que antes, por desconocimiento, no estaban integrados técnicamente en ningún guion.

A través del trabajo colaborativo se ha conseguido el cumplimiento de varias acciones hasta llegar a la formulación del documento dossier Proyec- 
to Geoparque Imbabura para su postulación ante la UNESCO, el mismo que fue presentado en su versión final, el día 7 de febrero del 2018.

\section{Materiales y métodos}

El presente proyecto es un estudio científico social que abarca las ciencias humanas y las ciencias de la Tierra, las ciencias humanas porque estas no sólo son aquellas ciencias o disciplinas científicas que se ocupan de aspectos del comportamiento y actividades de los seres humanos, sino todo lo que se relaciona con su accionar individual y colectivo; y, las ciencias de la Tierra o Geociencias por ser "las disciplinas de las ciencias naturales que estudian la estructura, morfología, evolución y dinámica del planeta Tierra" ECURED (2018). Como menciona Ciencias de la Tierra (2018) "constituyen una herramienta para planear una explotación racional de los recursos naturales, comprender las causas que originan los fenómenos naturales que afectan al ser humano y cómo éste influye en la naturaleza con sus acciones". La metodología de trabajo que se ha desarrollado es el diagnóstico investigativo urbano y rural participativo, que consiste en un conjunto de técnicas basadas en el proceso de las interrelaciones en los fenómenos y no solamente en los hechos.

Esta investigación combinó los enfoques cuantitativo y cualitativo en diferentes etapas del estudio. El método cualitativo se caracteriza por estudiar la manera en que las personas interpretan su realidad social a partir de su relación con ella (Denzin, 1989). Los conocimientos fueron sistematizados a través de la investigación bibliográfica realizada durante todo el proceso de investigación e incluyó libros, tesis, disertaciones y artículos, páginas webs: Instituto Nacional de investigaciones Geológico Minero y Metalúrgico (INEGEMM), Instituto Nacional de Biodiversidad (INABIO), Indicadores UNESCO. Se utilizaron herramientas como grabadoras para recoger los testimonios de la población abordada; drones para documentar los geositios; fichas de observación tipo matricial que sirvieron para recoger hechos, objetivos, actitudes y opiniones de los sujetos estudiados; cámara fotográfica profesional para levantar la base de datos fotográficos del GPI, cuestionarios aplicados a líderes comunitarios.

Al ser una investigación cualitativa, estudio de caso de tipo acción-participativa, la metodo- logía de trabajo que se ha desarrollado es el diagnóstico investigativo urbano y rural, este es un método cualitativo por excelencia, pero no hay que olvidar los datos cuantitativos para una mejor comprensión del problema de investigación, por tanto la investigación participativa consiste en un conjunto de técnicas basadas en el proceso de las interrelaciones en los fenómenos y no solamente en los hechos. Primero se delimitaron unos objetivos, después se elaboró un diagnóstico y se recogieron posibles propuestas que salgan de la propia praxis participativa y que puedan servir de base para su debate y negociación entre todos los participantes. Finalmente, las propuestas se concretan en líneas de actuación, donde los sectores implicados asumen un papel protagonista en el desarrollo del proceso. En el trabajo empírico, la recolección de datos se basó en la observación participante la cual sirvió para identificar las vocaciones productivas y culturales en cada uno de los seis cantones del PGI, actividades cotidianas, rituales, interacciones, y eventos de la gente que es estudiada como uno de los medios para aprender sobre su cultura.

\section{Resultados}

El trabajo mancomunado y colaborativo de los entes involucrados en el proyecto ha permitido alcanzar ciertos resultados (R) que se encuadran dentro los objetivos específicos planteados al inicio; entre los más relevantes están:

\subsection{R1. Diseño esquemático de la estrategia para procurar un desarrollo territorial sustentable, teniendo como eje al Proyecto Geoparque Imbabura.}

Se inicia con el reconocimiento de que el territorio de Imbabura, es parte de una nación, de un Estado, de un país llamado Ecuador; y que es afectado de forma positiva o negativa conforme a las decisiones del poder central y a las condiciones de su entorno, a nivel nacional e internacional (Figura 1). 


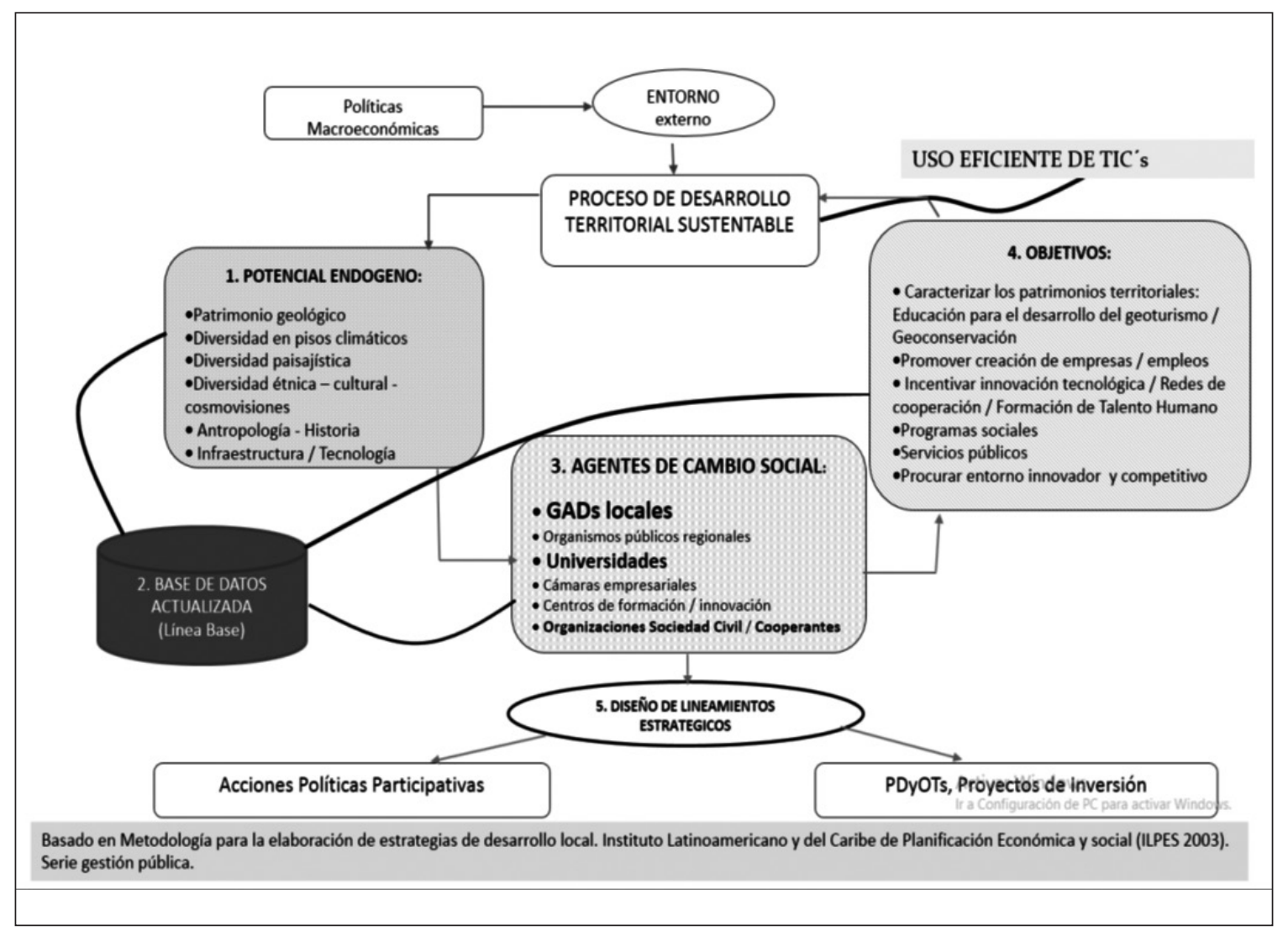

Figura 1. Resumen esquemático de la estrategia para el desarrollo local (Comité de Gestión Proyecto Geoparque Imbabura, 2017).

Este proceso contribuirá al posicionamiento de Imbabura y Ecuador a nivel nacional e internacional. En este marco, como resultados preliminares, se derivarán nuevos proyectos, desde diferentes perspectivas para desarrollar los componentes esenciales; a saber: educación, geoturismo y conservación con una filosofía de desarrollo local sustentable. Se trata entonces, de desarrollar competencias con la intervención activa de sus propios actores locales, con abundancia de mente y generosidad para unir esfuerzos, conocimientos, experiencias; es decir, la cohesión social de todos los involucrados es esencial.

\subsection{R2. Articulación del Comité de Gestión y los amigos del Proyecto Geoparque Imbabura}

Fue uno de los puntos de partida para empezar con el desarrollo del proyecto, los componentes esenciales de valor del Proyecto Geoparque Imbabura son educación, geoturismo y conservación. El equipo de trabajo, articulado en el Comité de Gestión, con sentido de pertenencia y pertinencia, vocación de servicio, forjando buenas prácticas de trabajo colaborativo interinstitucional, conforme a lo dispuesto por el Consejo Provincial de Imbabura, se propuso promover este proceso desde la acción comunitaria, poniendo en práctica la "MINGA" como valor cultural local de trabajo en equipo, en un sentido de abajo hacia arriba, socializando la propuesta en todos los ámbitos del territorio imbabureño, de tal manera que paso a paso, la población se empodere con la causa (Figura 2). Desde el año 2016, se mantiene activa la campaña "AMIGOS del Proyecto Geoparque Imbabura", a través de la cual, se generan espacios para la socialización y reflexión respecto a la necesidad e importancia de desarrollar valores de responsabilidad social y participación activa en el proceso de desarrollo local (Comité de Gestión Proyecto Geoparque Imbabura, 2017).

Este proceso contribuirá al posicionamiento de Imbabura y Ecuador a nivel nacional e internacional. En este marco, como resultados preliminares, se derivarán nuevos proyectos, desde diferentes perspectivas para desarrollar los componentes esenciales; a saber: educación, geoturismo y conservación con una filosofía de desarrollo local sustentable. Se trata entonces, de desarrollar competencias con la intervención activa de sus propios actores locales, con abundancia de mente y generosidad para unir esfuerzos, conocimientos, experiencias; es decir, la cohesión social de todos los involucrados es esencial. 


\subsection{R2. Articulación del Comité de Gestión y los amigos del Proyecto Geoparque Imbabura}

Fue uno de los puntos de partida para empezar con el desarrollo del proyecto, los componentes esenciales de valor del Proyecto Geoparque Imbabura son educación, geoturismo y conservación. El equipo de trabajo, articulado en el Comité de Gestión, con sentido de pertenencia y pertinencia, vocación de servicio, forjando buenas prácticas de trabajo colaborativo interinstitucional, conforme a lo dispuesto por el Consejo Provincial de Imbabura, se propuso promover este proceso desde la acción co- munitaria, poniendo en práctica la "MINGA" como valor cultural local de trabajo en equipo, en un sentido de abajo hacia arriba, socializando la propuesta en todos los ámbitos del territorio imbabureño, de tal manera que paso a paso, la población se empodere con la causa (Figura 2). Desde el año 2016, se mantiene activa la campaña "AMIGOS del Proyecto Geoparque Imbabura", a través de la cual, se generan espacios para la socialización y reflexión respecto a la necesidad e importancia de desarrollar valores de responsabilidad social y participación activa en el proceso de desarrollo local (Comité de Gestión Proyecto Geoparque Imbabura, 2017).

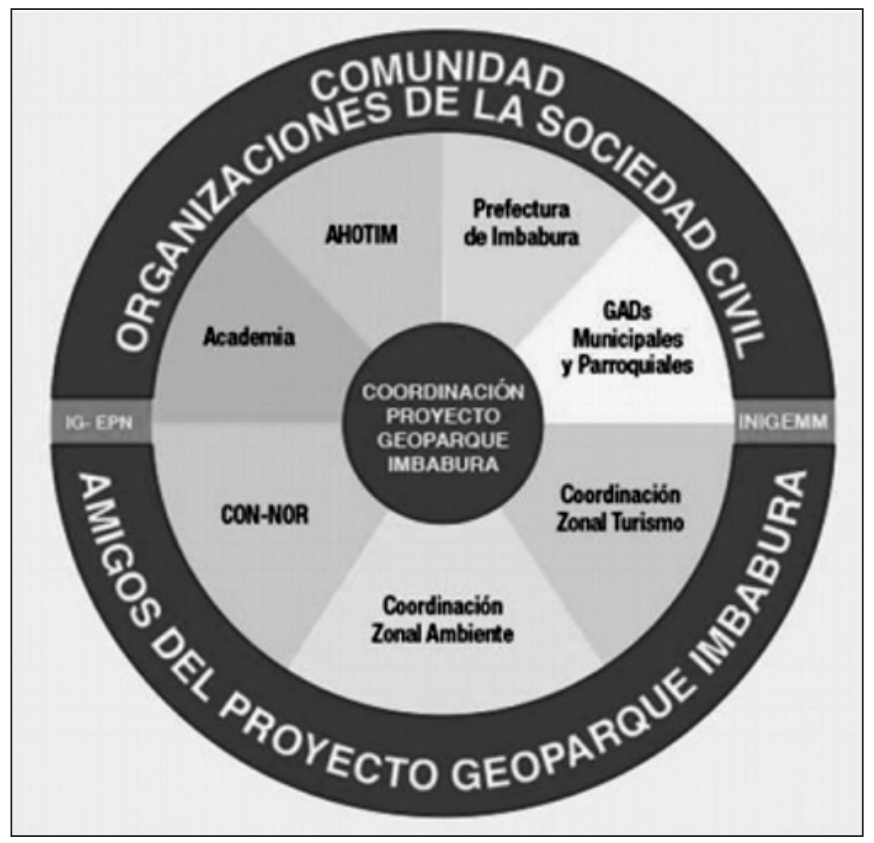

Figura 2. Esquema de articulación del Comité de Gestión y los amigos del Proyecto Geoparque Imbabura (Comité de Gestión Proyecto Geoparque Imbabura, 2017).

\subsection{R3. Análisis del potencial geoturístico}

La provincia de Imbabura, cuyo territorio es propuesto como aspirante a la denominación Geoparque Mundial de la UNESCO, es reconocida por la belleza de su paisaje, por la presencia de al menos once centros volcánicos y lagos o lagunas y cascadas en cada uno de sus cantones. En pocos minutos es posible pasar del frío de los páramos al clima abrigado de sus valles, pasando por un estado primaveral en los centros urbanos. Los volcanes de Imbabura, datados en el período geológico Cuaternario están clasificados como apagados, potencialmente activos y activos; son atractivos para la investigación científica. Desde los miradores del entorno, es propicio explicar su evolución y comportamiento tomando como base la información proporcionada por los investigadores del Instituto Geofísico de la Escuela Politécnica Nacional (IG-EPN) y del Instituto de Investigación Geológico Minero y Metalúrgico (INIGEMM). Estas instituciones son entidades técnicas-científicas AMIGAS del Proyecto Geoparque Imbabura.

Prácticamente en todos los geositios están trazados senderos, susceptibles de identificar lugares de interés para dar explicación y agregar valor a la actividad turística y transformarla en geoturismo. El funcionamiento de las fuentes de agua ancestrales, cascadas y lagunas es otra atracción. En las faldas del complejo volcánico Imbabura, es visible lo mágico de la naturaleza, se aprecia el nacimiento de las fuentes de agua fresca desde las profundidades de la tierra, para ser utilizadas como agua de riego para los cultivos, agua 
potable para los hogares y alimentación natural para el lago San Pablo. Más adelante el volumen de agua de las vertientes toma mayor dimensión cuando caen como desfogue por la cascada de Peguche.

Desde la cosmovisión de los pueblos originarios, estos geositios tienen relevancia por ser considerados sagrados y son lugar de los rituales de purificación según sus propias costumbres. En la zona de Íntag y en Urcuquí la presencia de aguas termales, la participación de la Corporación Eléctrica del Ecuador, la Agencia de Cooperación del Japón (JICA), las informaciones geológicas que estas instituciones generan en la exploración geotérmica, los actores locales y los flujos turísticos, hacen pensar en la posibilidad de emprender en nuevos medios de vida para las comunidades cercanas.

En medio de este relieve y geografía, con pisos altos y bajos, entre volcanes, valles y lagunas, por los caminos de Imbabura, es frecuente ver a grupos organizados de deportistas recreativos en las disciplinas de trekking y ciclismo de montaña, disfrutando del entorno natural del Proyecto Geoparque Imbabura. Varios de estos grupos, respondiendo a la invitación de la campaña AMIGOS, ya se han acercado para participar en el proceso de fundamentación y difusión de la causa IMBABURA "Geoparque Mundial de la UNESCO". Todo esto se fortalece de forma vital con el encuentro cotidiano de los pueblos ancestrales andinos, afroecua- torianos y mestizos; esta cultura VIVA hace que orgullosamente en Imbabura se promueva valores de interculturalidad en reconocimiento de las cosmovisiones, costumbres y tradiciones de sus pueblos.

\subsection{R4. Listado y descripción de sitios geológicos dentro del geoparque propuesto}

Un geositio como mencionan Carcavilla Urquí \& Cortés (2018) llamado también lugar de interés geológico (LIG) "es un área que forma parte del patrimonio geológico de una región natural por mostrar, de manera continua en el espacio, una o varias características consideradas de importancia en la historia geológica de la misma.

Imbabura ha desarrollado una descripción geológica general del geoparque, en la cual se identificaron principalmente tres tipos de suelo: entisoles, inceptisoles y molisoles, y además se reconoció once centros volcánicos en la provincia, los cuales están ubicados tanto en el Valle Interandino, como en la Cordillera Occidental. Se procedió a organizar la información recolectada en tablas, por cada uno de los seis cantones de la provincia de Imbabura: Ibarra, Otavalo, Cotacachi, Antonio Ante, Urcuquí y Pimampiro, para organizar los geositios. A continuación, en la tabla 1 se muestran los geositios del cantón Ibarra.

Tabla 1. Listado y descripción de sitios geológicos dentro del geoparque propuesto (Fuente: Dossier Proyecto Geoparque Imbabura 2017)

\begin{tabular}{|c|c|c|c|}
\hline $\mathbf{N}^{\circ}$ & Nombre & Tipo & Estado \\
\hline IB01 & Taita Imbabura & Volcán & $\begin{array}{l}0.260680,-78.179662 ; 4.624 \mathrm{~m}, \\
\text { potencialmente activo }\end{array}$ \\
\hline IB02 & Huarmi Imbabura & Volcán & $\begin{array}{l}0.234037,-78.192388 ; 3.840 \mathrm{~m}, \\
\text { potencialmente activo }\end{array}$ \\
\hline IB03 & Cubilche & Volcán & Volcán extinto \\
\hline IB04 & Cunrru & Volcán & Volcán extinto \\
\hline IB05 & Artezón & Domo & \\
\hline IB06 & Angaraloma & Domo & \\
\hline IB07 & Cordillera Angochagua & Montañas & \\
\hline IB08 & La Esperanza & Páramo & \\
\hline IB09 & Yahuarcocha & Laguna & \\
\hline IB10 & Cubilche lake & Laguna en caldera de volcán & \\
\hline IB11 & Guayabillas & Bosque protector & \\
\hline IB12 & La Rinconada & Valle & \\
\hline IB13 & Chota & Valle & \\
\hline IB14 & Salinas & Valle & \\
\hline IB15 & Añaspamba & Páramo & \\
\hline IB16 & Zuleta & Páramo & \\
\hline
\end{tabular}




\begin{tabular}{|l|l|l|l|}
\hline \multicolumn{3}{|c|}{ Geositios del cantón Otavalo } \\
\hline No. & Nombre & Tipo & Estado \\
\hline OT01 & Mojanda & Complejo volcánico & Volcán extinto \\
\hline OT02 & Fuya Fuya & Volcán & Volcán extinto \\
\hline OT03 & Cushnirumi & Volcán & Volcán extinto \\
\hline OT04 & San Jorge & Domo & \\
\hline OT05 & Colangal & Domo & \\
\hline OT06 & San Pablo & Laguna & \\
\hline OT07 & Lagunas de Mojanda (Caricocha, & Complejo de lagunas & \\
\hline OT08 & Cascada de Peguche & Cascada & \\
\hline OT09 & Bosque Protector Peguche & Bosque protector & \\
\hline OT10 & Cascada taxopamba & Forest & \\
\hline
\end{tabular}

Figura 3. Geositios del cantón Otavalo (Comité de Gestión Proyecto Geoparque Imbabura, 2017).

\begin{tabular}{|l|l|l|l|}
\hline \multicolumn{3}{|c|}{ Geositios del cantón Cotacachi } \\
\hline No. & Nombre & Tipo & Estado \\
\hline CO01 & Cotacachi & Estrato volcán & Volcán Extinto \\
\hline CO02 & Cuicocha & $\begin{array}{l}\text { Volcán y Laguna en } \\
\text { caldera }\end{array}$ & Volcán active \\
\hline CO03 & $\begin{array}{l}\text { Reserva Ecológica Cotacachi - } \\
\text { Cayapas }\end{array}$ & Reserva natural & \\
\hline CO04 & Intag & Valle & Hotspost \\
\hline CO05 & Irubi & Valle & \\
\hline CO06 & Cara del Dios de Intag & Geomorfología & \\
\hline CO07 & Rio Intag & Rio & \\
\hline CO08 & Bio- corredor del Chocó & $\begin{array}{l}\text { Ecosistema forestall - } \\
\text { bosque primario }\end{array}$ & \\
\hline CO09 & Termas de Nangulvi & Aguas termales & \\
\hline
\end{tabular}

Figura 4. Geositios del cantón Cotacachi (Comité de Gestión Proyecto Geoparque Imbabura, 2017).

\begin{tabular}{|l|l|l|l|}
\hline \multicolumn{2}{|c|}{ Geositios del cantón Urcuquí } \\
\hline No. & Nombre & Tipo & Estado \\
\hline UR01 & Yanaurcu de Piñán & Volcán & \\
\hline UR02 & Chachimbiro & Complejo volcánico & Potencialmente active \\
\hline UR03 & La Viuda & Domo & \\
\hline UR04 & Cochapata & Domo & \\
\hline UR05 & Hugá & Domo & \\
\hline UR06 & Tanguna & Domo & \\
\hline UR07 & Tababara & Domo & \\
\hline UR08 & Laguna Tobar Donoso & Laguna & \\
\hline UR09 & Complejo lacustre de Piñan & Complejo de lagunas & \\
\hline UR10 & Termas Chachimbiro & Aguas termales & \\
\hline UR11 & Termas de Timbuyacu & Aguas termales & \\
\hline UR12 & Termas Arco Iris & Aguas termales & \\
\hline UR13 & Cascada Conrayaro & springs & \\
\hline UR14 & Cascada Condor Paccha & Cascada & \\
\hline UR15 & Cascada Nido del Condor & Cascada & \\
\hline UR16 & Páramos de Piñan & Cascada & \\
\hline
\end{tabular}

Figura 5. Geositios del cantón Urcuquí (Comité de Gestión Proyecto Geoparque Imbabura, 2017). 


\begin{tabular}{|l|l|l|l|}
\hline \multicolumn{4}{|c|}{ Geositios del cantón Pimampiro } \\
\hline No. & Nombre & Tipo & Estado \\
\hline PI01 & Coordillera Pimampiro & Montañas & \\
\hline PI02 & Laguna de Puruhanta & Laguna & \\
\hline PI03 & Reserva Cayambe - Coca & Reserva natural & \\
\hline PI04 & Laguna Negra & Laguna & \\
\hline PI05 & Cascada El Arenal & Cascada & \\
\hline
\end{tabular}

Figura 6. Geositios del cantón Pimampiro (Comité de Gestión Proyecto Geoparque Imbabura, 2017)

Todo ello forma parte del patrimonio geológico que posee la provincia de Imbabura lo que la hace potencialmente atractiva para el desarrollo de actividades turísticas, lo cual beneficiará al desarrollo económico-turístico de las comunidades del área de influencia.

\subsection{R5. Instalaciones existentes y planificadas para el geoparque Imbabura}

Imbabura cuenta con accesos por la parte sur desde la capital de la República y norte, desde la provincia del Carchi y Colombia, de primera clase; la del sur es de tres carriles en cada sentido y las del norte, de dos carriles por sentido. Desde la provincia de Esmeraldas, por el occidente, la vía se mantiene en buenas condiciones con un carril por sentido. La conectividad entre cantones es rápida. En cuanto a instalaciones, en Otavalo, está el Instituto Otavaleño de Antropología; en Cotacachi, el Museo de las Artes y el Centro de Interpretación Geológico y Natural de la Reserva Ecológica Cotacachi-Cayapas; en Antonio Ante, sobresale la fábrica Imbabura; en Ibarra, el Centro Cultural "El Cuartel", el museo arqueológico del Banco Central del Ecuador.

Están en desarrollo, obras como el boulevard en la cuenca del lago Yahuarcocha y el nuevo muelle a orillas del lago San Pablo. Esta nueva infraestructura, seguramente, potenciará y mejorará la calidad de los servicios turísticos en estos geositios. Factores importantes, serán los procesos de capacitación y educación para mejorar la conducta de los operadores de turismo (geoturismo) y servicios gastronómicos en su relación con el entorno para procurar buenas prácticas de geoconservación.

Como se nota en el detalle de esta sección, hace falta la implementación de medios de difusión y comunicación relacionados al posicionamiento del Proyecto Geoparque Imbabura. En este sentido, es tarea fundamental del Comité de Gestión, impulsar la instalación de paneles informativos pertinentes a la descripción de cada uno de los geositios y centros de interpretación didácticos que faciliten la comprensión de la evolución geológica de la Tierra en el territorio del Proyecto Geoparque Imbabura.

\subsection{R6. Actividad económica y plan comercial}

Para desarrollar este tema, se recurre a la identificación de las vocaciones productivas en cada uno de los cantones en el área del Proyecto Geoparque Imbabura. Así, Otavalo es la puerta de entrada desde Quito, se caracteriza por ser un polo de atracción turística, especialmente por la presencia del "indio otavaleño", su vestimenta típica, sus costumbres y tradiciones. Aquí, está la feria artesanal más grande de América del Sur. La Plaza de Ponchos, es el eje del comercio cantonal. La cercanía de geositios con valor geológico y paisajístico ha motivado la implementación de emprendimientos turísticos, prácticamente en todo el cantón.

En Cotacachi, la característica principal es la industria del cuero, se ofrece una variada gama de productos, desde artesanía, souvenirs y prendas de vestir; en la parte andina, sobresale la presencia del complejo volcánico Cotacachi-Cuicocha y la Reserva Natural Cotacachi-Cayapas, en la parte más occidental está la zona de Íntag con atributos diferentes; es un valle rodeado de montañas, con características geológicas muy importantes, tiene minas de cobre, oro, mármol y canteras de donde se extrae la materia prima para la producción de cemento; los productos alimenticios y frutas subtropicales no faltan; todos estos argumentos hacen que Cotacachi también tenga una muy buena atracción para turistas nacionales y extranjeros.

En Antonio Ante está presente de forma predominante la industria textil, su ícono principal es la fábrica Imbabura, su edificio, parte del patrimonio de la ciudad, ahora está convertido en museo; Atuntaqui satisface la demanda de prendas de vestir en un porcentaje importante del mercado ecuatoriano; se ha- 
cen esfuerzos por ingresar al mercado internacional con exportaciones textiles, cuyos indicadores, cada vez alcanzan niveles de mayor competitividad; otro atributo de Antonio ante, es la gastronomía, todos los fines de semana, especialmente, se presencian flujos importantes de turistas que viene a saborear su deliciosa fritada (carne de cerdo frita en su propia grasa, aderezada con varias especias).

Ibarra es la capital provincial, aquí se asientan las delegaciones del Gobierno nacional y se constituye en el principal centro comercial de Imbabura; tiene una importante oferta de productos gastronómicos y su cercanía a geositios de especial paisaje, más las vías en buen estado facilitan la presencia de importantes flujos turísticos regionales, especialmente procedentes de la capital de la República. El volcán Taita Imbabura tiene visibilidad prácticamente desde todos los cantones. Su ubicación es estratégica y es el ícono natural de la provincia. En torno a este, más los demás geositios y sitios de atracción, el Tren Ecuador recientemente rehabilitado es una atracción; recorre una ruta que pasa por puentes y túneles construidos a inicios del siglo anterior y une comunidades indígenas, mestizas y afroecuatorianas. En los valles de Salinas y del Chota, predominan los cultivos de caña de azúcar. En Ibarra se destacan los bordados a mano de Zuleta, en la parroquia Angochagua, y los tallados artesanales en madera de San Antonio. En la parte urbana, los "helados de paila" con tradición centenaria, son delicias que el turista siempre llega a degustar.

Urcuquí, cantón ubicado a 30 minutos de Ibarra, hacia el noroeste, es reconocido por la calidad de sus aguas termales. Hasta sus balnearios llegan turistas de todo el país y de los departamentos del sur de Colombia. En este cantón se desarrolla la "Ciudad del Conocimiento" con su Universidad Yachay Tech, que se espera, se convierta en el laboratorio que permita desarrollar procesos, metodología y tecnologías para agregar valor a la producción local y nacional con la finalidad de mejorar el nivel de competitividad mediante el cambio de la matriz productiva; en Urcuquí, en este momento se está desarrollando la exploración y estudios para evaluar la factibilidad de generar energía geotérmica.

Pimampiro, ubicado en la parte oriental del Proyecto Geoparque Imbabura, desde hace décadas es el granero de productos agrícolas; su población es laboriosa y dedicada ahora al cultivo de frutales, verduras y hortalizas; desde sus campos se abastecen los mercados de toda la provincia y los intermediarios realizan sus ofertas de compra para llevar la producción hasta el centro del país. Recientemente en Pimampiro se reconoció a una de sus costumbres tradicionales, "el trueque", como parte del patrimonio intangible del Ecuador.

Sin embargo, de lo detallado en el aspecto económico, hasta hace poco tiempo, la gestión de desarrollo se la hacía de forma independiente desde cada uno de los cantones, de forma desarticulada; en los últimos años, las autoridades se han puesto de acuerdo para gestionar la provincia como un solo territorio, fortaleciendo las capacidades locales.

El Proyecto Geoparque Imbabura, se ha convertido en el eje articulador e instrumento para desarrollar el territorio de forma integral, articulando esfuerzos entre todos los actores locales, tratando de poner en valor sus patrimonios endógenos. La conformación de su Comité de Gestión, su relación con la comunidad, las nuevas tendencias sociales amigables con el ambiente, son cada vez más cercanas y hace que el camino hacia un desarrollo local sustentable sea viable.

\subsection{R7. Políticas para el desarrollo sostenible de geoturismo y economía, geoeducación y geo- patrimonio.}

El inicio del proceso del Proyecto Geoparque Imbabura, coincide con la declaración desde $\mathrm{Na}$ ciones Unidas, para impulsar en todo el mundo los Objetivos de Desarrollo Sostenible. Este marco dio sentido, con dimensión global, a los esfuerzos por incluir en la formación de los nuevos profesionales, los criterios para caminar hacia un desarrollo territorial sustentable. En Imbabura, fue la Universidad Técnica del Norte la pionera en ofrecer un programa de formación académica de cuarto nivel relacionada con esta temática: Maestría en Recursos Naturales Renovables, mención Manejo y Conservación de Suelos (primera cohorte) donde el nuevo enfoque de desarrollo económico tenía íntima relación con el cuidado del ambiente y con la conciencia social.

Antes, Ban Ki-moon, en su informe de fin de período como Secretario General de las Naciones Unidas, haciendo un balance sobre los avances en el cumplimiento de metas de los Objetivos del Milenio, informaba que, "aunque se habían logrado progresos importantes en la erradicación de la pobreza extrema, la protección del medio ambiente y la generación de oportunidades para todos", aún había mucho por hacer. En este sentido, como política institucional, el Proyecto Geoparque Imbabura, impulsará sus acciones en concordancia con los Objetivos de Desarrollo Sostenible, con los artículos pertinentes a los derechos de la Pacha Mama, consagrados en la Constitución del Ecuador y en el Programa de Gobierno de la Prefectura de Imbabura. Con este propó- 
sito, el Comité de Gestión ha sido conformado con los representantes de los actores locales, para que exista participación amplia, con aportes y sentido crítico de la realidad y del escenario prospectivo al que nos queremos dirigir.

Así, en el Proyecto Geoparque Imbabura, la función Geoeducación está ubicada en primer lugar, porque es fundamental inculcar en la población a través del conocimiento del entorno, una conducta amigable con el ambiente; la articulación de actores locales, públicos y privados del sector rural y urbano, paso a paso va generando sinergias; el espíritu emprendedor de la población se evidencia en la apertura de medios de vida individuales y asociativos que evidencian una mejor calidad de vida para toda la comunidad.

\subsection{R8. Políticas y ejemplos del empoderamiento de la comunidad}

Para noviembre 30 de 2015, el Comité de Gestión del Proyecto Geoparque Imbabura, había hecho una convocatoria pública, como política de "participación ciudadana", para recibir propuestas de logotipos, de entre los cuales, mediante un jurado independiente se seleccionaría la imagen oficial. La respuesta fue muy buena; se recibieron 57 propuestas y de ellas, efectivamente se seleccionó la imagen oficial del Proyecto Geoparque Imbabura; en el programa de premiación a los ganadores, hubo casa llena. En cumplimiento de esta misma política, de participación ciudadana, se pone en ejecución, de forma permanente, la campaña "AMIGOS" del Proyecto Geoparque Imbabura; mediante este instrumento personas naturales, jurídicas, públicas y privadas, organizaciones de la sociedad civil, presentan sus adhesiones al proyecto y presentan su predisposición para trabajar juntos por el fortalecimiento de la identidad provincial compartiendo la ilusión de alcanzar la denominación "Geoparque Mundial de la UNESCO". Actualmente, las adhesiones son frecuentes y el interés por ser parte del proceso es evidente; las actividades que se realizan son de carácter educativo y formativo, recreativo, deportivo, ambiental; inspiradoras para fomentar el espíritu emprendedor desde el geoturismo.

Luego, como política de "trabajo articulado y colaborativo", se establecen alianzas y se procura el trabajo en red. La estructura del Comité de Gestión, es una evidencia de la visión compartida por mejores días para toda la provincia de Imbabura; el contacto con los aspirantes Geoparque Volcán Tungurahua y Napo-Sumaco, en Ecuador, es cercana y familiar, como producto de esta política, las instituciones téc- nicas-científicas afines al proyecto, cooperan con sus conocimientos para fundamentar la propuesta y como resultado poco a poco mediante actividades de socialización y comunicación, la comunidad está mejor informada y se empodera con la iniciativa.

El "Día Latinoamericano del Geoturismo", se celebró en abril de 2017 en la parroquia Angochagua, provincia de Imbabura; en la misma se demostró el trabajo articulado y colaborativo; se realizaron eventos de ciclismo, escalada, senderismo, charlas académicas entre otras actividades; a la coordinación de Grutas del Palacio en Uruguay, se envió oportunamente, en resumen, la variedad de actividades desarrolladas, donde se nota el nivel de participación de la comunidad.

\subsection{R9. Políticas de fortalecimiento del Proyecto Geoparque Imbabura}

Como política de "fortalecimiento de la conciencia social", de forma articulada con entidades "amigas", se han realizado programas para la celebración por el día del ambiente, del agua, turismo sostenible, propiedad intelectual para el desarrollo del turismo y la cultura, entre otros; las actividades son variadas, en campo o en aula, con la participación de niños, adolescentes, jóvenes, adultos, hombres y mujeres. La empresa privada, antes alejada de las entidades públicas, cada vez da mejores muestras de querer trabajar en forma coordinada. En la actualidad en la academia se trabaja en la planificación de proyectos de creación de geoproductos como: ají volcánico, jabones artesanales, entre otros, para poner en valor los recursos disponibles.

\subsection{R.10 Interés y argumentos para convertirse en un Geoparque MundiaL de la UNESCO}

Desde el año 2015, cuando se empezó a gestionar el Proyecto Geoparque Imbabura, uno de los resultados que se ha evidenciado es que la causa "IMBABURA, aspirante a Geoparque Mundial de la UNESCO", se ha convertido en eje articulador para el desarrollo local. Al interior de la Prefectura de Imbabura como institución patrocinadora principal, sus unidades técnicas y administrativas, las autoridades, lo han asimilado como un proyecto emblemático para la cohesión del territorio y se han identificado productos y resultados que sirven como evidencia de gestión y trabajo colaborativo.

En la comunidad, las unidades educativas, las organizaciones de la sociedad civil, han recibido con beneplácito la propuesta y se suman con sus propios roles para participar activamente en el proceso. La 
conformación del Comité de Gestión con representantes de los gobiernos locales, de las entidades desconcentradas del Gobierno nacional, del sector empresarial y la academia, da transparencia al proceso y es un espacio propicio para generar sinergias e identificar oportunidades de acción de forma conjunta.

La campaña AMIGOS, puesta en marcha para incorporar adhesiones al proyecto, muestra como resultado, más de cuarenta entidades, entre públicas y privadas. Ellas participan con información, con actividades de campo, con capacitación, e inclusive con financiamiento en ciertas acciones de su interés.

Imbabura quiere formar parte de los geoparques mundiales de la UNESCO, porque este territorio tiene todo para convertirse en uno de ellos. El Proyecto Geoparque Imbabura, se ha constituido en el instrumento fundamental para el desarrollo local; con una identidad provincial fuerte, intercultural, saludable, con bienestar, caracterizado porque sus ciudadanos conozcan, valoren y tengan buenas prácticas de conservación, con un acertado manejo de la capacidad de carga de cada geositio, se busca optimizar el uso técnico de estos espacios que son propicios para el desarrollo de sus medios de vida. El PGI pretende desarrollar esta posibilidad, caminando junto a las familias de la zona de influencia, para generar oportunidades.

iLa visión de alcanzar para Imbabura, la denominación "Geoparque Mundial de la UNESCO”, es compromiso de todos! con ese lema, el comité de gestión del Proyecto Geoparque Imbabura trabaja comprometido y empoderado por el logro de esa denominación.

\subsection{R11. Formulación de documento dossier Pro- yecto Geoparque Imbabura para su postula- ción ante la UNESCO}

El año 2015 constituyó un punto de inflexión en el quehacer político, económico, social, deportivo, académico-científico de la provincia de Imbabura; fue la Prefectura de Imbabura la entidad que, a través de la Dirección de Cooperación Internacional, se acogió al PGP como proyecto de prioridad uno. Las primeras reuniones sirvieron para conocer el interés que generaba el proyecto en la sociedad; $y$, en especial, para socializar las observaciones que una Comisión Evaluadora de la UNESCO, había realizado a la primera presentación del dossier. Una vez conformado el Comité de Gestión del PGI se empezó a realizar una serie de actividades las cuales acogían las sugerencias de la comisión evaluadora.

Se organizaron cronogramas, mensuales, trimestrales, semestrales con actividades de diversa índole, todas las cuales fueron debidamente documentadas para empezar a conformar el nuevo dossier del proyecto. Cada acción debía estar debidamente articulada y aprobada por el comité de gestión, en pro de garantizar la rigurosidad técnica, científica, que demandaba el proyecto. Cada ente involucrado desde su ámbito, empezó a actuar en el territorio, lo cual generó una nueva forma de trabajo colaborativo más enfocado en el trabajo de campo que de oficina. Se manejó incluso el autofinanciamiento en ciertas acciones como adquisición de camisetas identificativas, premio para el ganador del logotipo del proyecto, salidas de campo, entre otros.

El dossier definitivo del Proyecto Geoparque Imbabura (Figura 7), postulante para obtener la denominación de Geoparque Mundial de la UNESCO, fue presentado el día 7 de febrero del 2018.

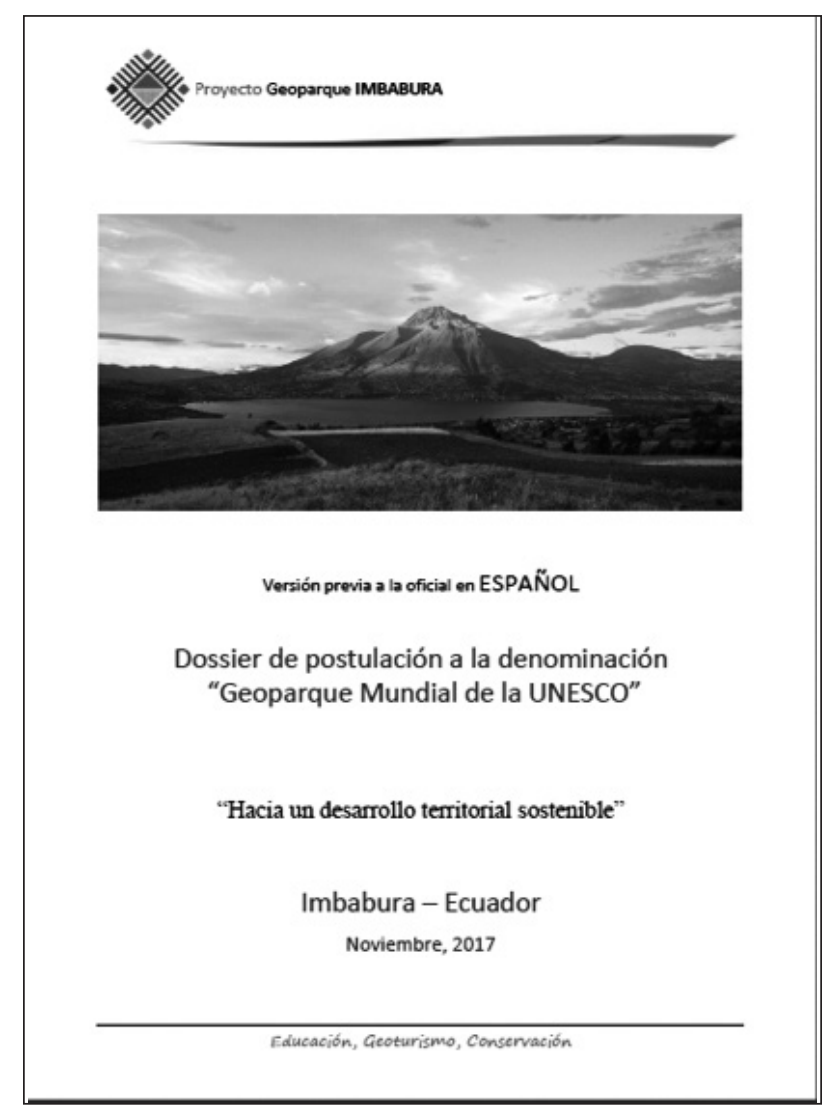

Figura 7. Portada del dossier del Proyecto Geoparque Imbabura (Comité de Gestión Proyecto Geoparque Imbabura, 2017).

\section{Discusión}

Un aspecto que se convierte en debilidad es la falta de conocimiento de la población de las características de su entorno natural. La gente se acostumbró a vivir en una provincia de paisaje hermoso, pero 
en su generalidad, no es capaz de describirla apropiadamente. Por esta razón, es que el componente Educación está ubicado en primer lugar entre las funciones a cumplir desde el geoparque propuesto. Se hace necesario, multiplicar esfuerzos, articulados entre los actores locales para desarrollar capacidades, mediante la investigación del entorno inmediato y sus valores culturales; la documentación, sistematización y socialización entre la población. Este componente será fundamental en el proceso de fortalecimiento de la identidad provincial.

Las características naturales del territorio en el geoparque propuesto, la diversidad de sus vocaciones productivas, la muy buena infraestructura vial, la ubicación tan cercana a Quito, capital de la República del Ecuador, y ser parte de la zona de frontera con Colombia, genera consecuencias por el fenómeno migratorio y de movilidad humana. Por un lado, positivos por la afluencia de turistas en busca de productos y destinos que contribuyen a la economía local. Esto demanda cada vez una oferta de servicios urbanos y rurales mejor cualificados. Esto es bueno, la organización, la creatividad y la innovación se ponen de manifiesto. Por otro lado, esta presión, genera secuelas negativas por la contaminación del ambiente y pueden poner en riesgo la seguridad habitual y la conservación de los geositios y sitios de interés, si no se implementa un plan integral con buenas prácticas para la gestión del territorio.

El potencial minero de la provincia de Imbabura es importante, por lo mismo, es oportuno que se tomen las medidas adecuadas para racionalizar su explotación de ser el caso; o, se planteen alternativas para el desarrollo económico y social amigables con el ambiente.

En Ecuador con fecha 2 de abril de 2017, se da inicio a una nueva administración del Gobierno nacional; con ello, se plantea el Plan Nacional de Desarrollo para Toda una Vida, período 2017-2021. Se estructura en tres ejes con sus propios objetivos articulados entre sí, con una filosofía de desarrollo territorial sustentable; a saber:

Eje 1. Derechos para todos durante toda la vida; Eje 2. Economía al servicio de la sociedad; Eje 3. Más sociedad, mejor Estado.

Los criterios del Proyecto Geoparque Imbabura, se identifican con el Plan Nacional, en cuanto procuran una vida digna con iguales oportunidades para todas las personas, la afirmación intercultural $y$ plurinacionalidad, revalorizando las identidades diversas; $\mathrm{y}$, precautelan los derechos de la natura- leza para las actuales y futuras generaciones. Al mismo tiempo, el PGI, será un instrumento para la gestión de cooperación internacional, precautelar la paz, la internacionalización de Imbabura y el posicionamiento del Ecuador en el mundo.

El Código Orgánico de Organización Territorial, Autonomía y Descentralización (COOTAD), es otro instrumento que da fundamento legal al PGI. El Art. 41, donde se refiere a las funciones del Gobierno Autónomo Descentralizado Provincial; dice, que una de ellas es: "Promover el desarrollo sustentable", en el marco de sus competencias constitucionales y legales. Complementariamente, el Programa de Gobierno de la Prefectura, está en congruencia con este marco legal.

Para poner en práctica este marco legal, la Prefectura de Imbabura, en trabajo conjunto con el Ministerio del Ambiente, Ministerio de Educación, los municipios, parroquias y comunidades, viene desarrollando cada año, un programa de gestión y educación ambiental, mediante el cual se involucra a unidades educativas, profesores y estudiantes en las acciones de conservación del ambiente para promover conciencia social y sentido de pertenencia con el territorio.

El Proyecto Socio Bosque, es una de las principales estrategias de conservación del patrimonio natural del Ecuador, protegiendo ecosistemas frágiles, refugio de especies amenazadas, por lo que fue considerado como parte de la meta 1 del objetivo 7 del Plan Nacional para el Buen Vivir 2013-2017, “Aumentar la proporción del territorio continental bajo conservación o manejo ambiental al 35.9\%". Socio Bosque, de la misma forma, es el principal mecanismo de implementación de las acciones REDD+ en Ecuador para el pago por resultados de deforestación evitada y distribución de beneficios, lo que le ha permitido ser una iniciativa bandera REDD+ en las Conferencias de las Partes (COP) de Cambio Climático de Cancún, Lima y París, así como en el Foro de Bosques de las Naciones Unidas.

A nivel social, es el único programa ambiental que ha beneficiado de forma directa a más de 187.000 (ciento ochenta y siete mil) personas de organizaciones de base y de las nacionalidades indígenas del país: Aí Cofán, Andwa, Awá, Achuar, Chachi, Kichwa, Sekopai, Shuar, Shiwiar, Sapara, Woaorani y de pueblos afroecuatorianos, montubios y mestizos a lo largo de todo el Ecuador continental. El país cuenta con un área de conservación bajo esta figura de conservación de 1.489.217,74 Ha. 


\begin{tabular}{||l|r|r|r|}
\hline Tipo Socio & Ha. Conservación & \multicolumn{1}{|c|}{ Total Beneficiarios } & \multicolumn{2}{c|}{$\begin{array}{c}\text { Convenios } \\
\text { Firmados }\end{array}$} \\
\hline COLECTIVO & $1.300 .913,24$ & 174.746 & 191 \\
\hline INDIVIDUAL & $166.037,48$ & 10.091 & 2.545 \\
\hline SOCIO MANGLAR & $22.267,02$ & 3.314 & 23 \\
\hline Total general & $\mathbf{1 . 4 8 9 . 2 1 7 , 7 4}$ & $\mathbf{1 8 8 . 1 5 1}$ & $\mathbf{2 . 7 5 9}$ \\
\hline
\end{tabular}

Figura 8. Área total en conservación nacional - Programa Socio Bosque (SEMOP-MAE, 2017).

Imbabura cuenta con 213 convenios, de los cuales 211 corresponden a socios individuales y 2 a socios colectivos, cubriendo un área de conservación de 19.319,33 has.

\begin{tabular}{|c|r|r|r|}
\hline Tipo Socio & Ha. Conservación & Total Beneficiarios & \multicolumn{1}{|c|}{$\begin{array}{c}\text { Convenios } \\
\text { Firmados }\end{array}$} \\
\hline COLECTIVO & $3,559,00$ & 1,290 & 2 \\
\hline INDIVIDUAL & 15759,50 & 773 & 211 \\
\hline Total general & 19319,33 & 2,063 & 213 \\
\hline
\end{tabular}

Figura 9. Área total en conservación provincia de Imbabura - Programa Socio Bosque (SEMOP-MAE, 2017).

Para procurar un desarrollo territorial sustentable, es imperativo trabajar en educación, en el fortalecimiento de la investigación aplicada y con pertinencia al territorio, en la difusión de los nuevos conocimientos, para generar oportunidades a partir del sentido de pertenencia e identificación con las potencialidades evidenciadas en la diversidad de sus patrimonios locales; a saber: geológico, natural, histórico, cultural e intercultural. De ahí, la trascendencia de ubicar a la educación como primer componente del Proyecto Geoparque Imbabura.

Por lo tanto, es necesario realizar acciones para propiciar que la comunidad local, en la práctica, sea capaz de concienciar, caracterizar, valorar, defender y conservar su propio entorno. Esta causa, invita a desarrollar capacidades y buenas prácticas en el aprovechamiento racional de sus recursos. El turismo que, desde la perspectiva geológica, pudiera denominarse geoturismo, es una de las vocaciones territoriales. Las instituciones y organizaciones de la sociedad civil con incidencia en la provincia; llámense: gobiernos locales, organismos públicos regionales, unidades educativas, academia, sector privado, cooperantes; desde la perspectiva del pro- yecto, serán vistas y entendidas desde ahora como entidades de cambio social, cada una desde su propio rol, participantes activas en el proceso de empoderamiento y del saber consciente que Imbabura es un territorio con las características de geoparque. La academia tiene una responsabilidad enorme al revisar sus mallas curriculares y los diseños de carreras, los cuales deben direccionarse en pro de satisfacer la demanda que la denominación como Geoparque Mundial generará. La Pontificia Universidad Católica, sede Ibarra, se encuentra rediseñando la carrera de turismo, lo cual claramente va enfocado en reforzar micro curricularmente temas como: geografía turística, desarrollo sustentable, guianza turística, emprendimiento, conservación de patrimonio; logrando alcanzar y desarrollar capacidades y habilidades de los estudiantes en cuanto al conocimiento de gestión de geoparques.

Este proceso ha significado una experiencia enriquecedora porque se ha aprendido a trabajar en equipo, como lo hacían nuestros ancestros en la llamada minga (reunión solidaria de amigos y vecinos para hacer algún trabajo en común, luego del cual comparten una generosa comida pagada 
por los beneficiarios), en torno a objetivos colectivos con beneficio para las presentes y futuras generaciones. Los efectos de esta forma de trabajo se evidenciarán en las capacidades locales, en su calidad de vida, en su modelo de desarrollo con criterios de sustentabilidad.

Imbabura está segura de conseguir la denominación como Geoparque Mundial de la UNESCO, porque el proyecto en sí ha sido capaz como ningún otro, de crear sinergias entre el quehacer público, privado, académico y de la sociedad civil de Imbabura, lo cual ha significado una oportunidad de desarrollo local. A partir del inicio del proyecto, las comunidades indígenas se ven con otros ojos; lo que antes era atraso, pobreza, discriminación, hoy se ha convertido en orgullo de su identidad, oportunidades de capacitación continua y manejo técnico de sus recursos naturales y culturales, buenas prácticas de conservación, capacidad de carga de los destinos turísticos, recuperación de costumbres ancestrales con fines identitarios, entre otros. La parte urbana también tiene expectativas en mejorar la calidad de vida a través del manejo de flujos turísticos, venta de artesanías, dinamización del transporte, posicionamiento de nuestra gastronomía, innovación de oferta académica por parte de las IES, entre otras.

\section{Conclusiones}

Desde el año 2015, cuando se empezó a gestionar el Proyecto Geoparque Imbabura, se ha logrado que éste se convierta en eje articulador para el desarrollo local al interior de la Prefectura de Imbabura, como institución patrocinadora principal; sus unidades técnicas y administrativas, las autoridades, lo asimilaron como un proyecto emblemático para la cohesión del territorio y se han identificado productos y resultados que sirven como evidencia de gestión y trabajo colaborativo.

La conformación del Comité de Gestión con representantes de los gobiernos locales, de las entidades desconcentradas del gobierno nacional, del sector empresarial y la academia, da transparencia al proceso y es un espacio propicio para generar sinergias e identificar oportunidades de acción de forma conjunta. La campaña AMIGOS (Figura 10), puesta en marcha para incorporar adhesiones al proyecto, muestra como resultado, más de cuarenta entidades, entre públicas y privadas. Ellas participan con información, actividades de campo, capacitación, inclusive con financiamiento en ciertas acciones de su interés.

La estructura del Comité de Gestión, es una

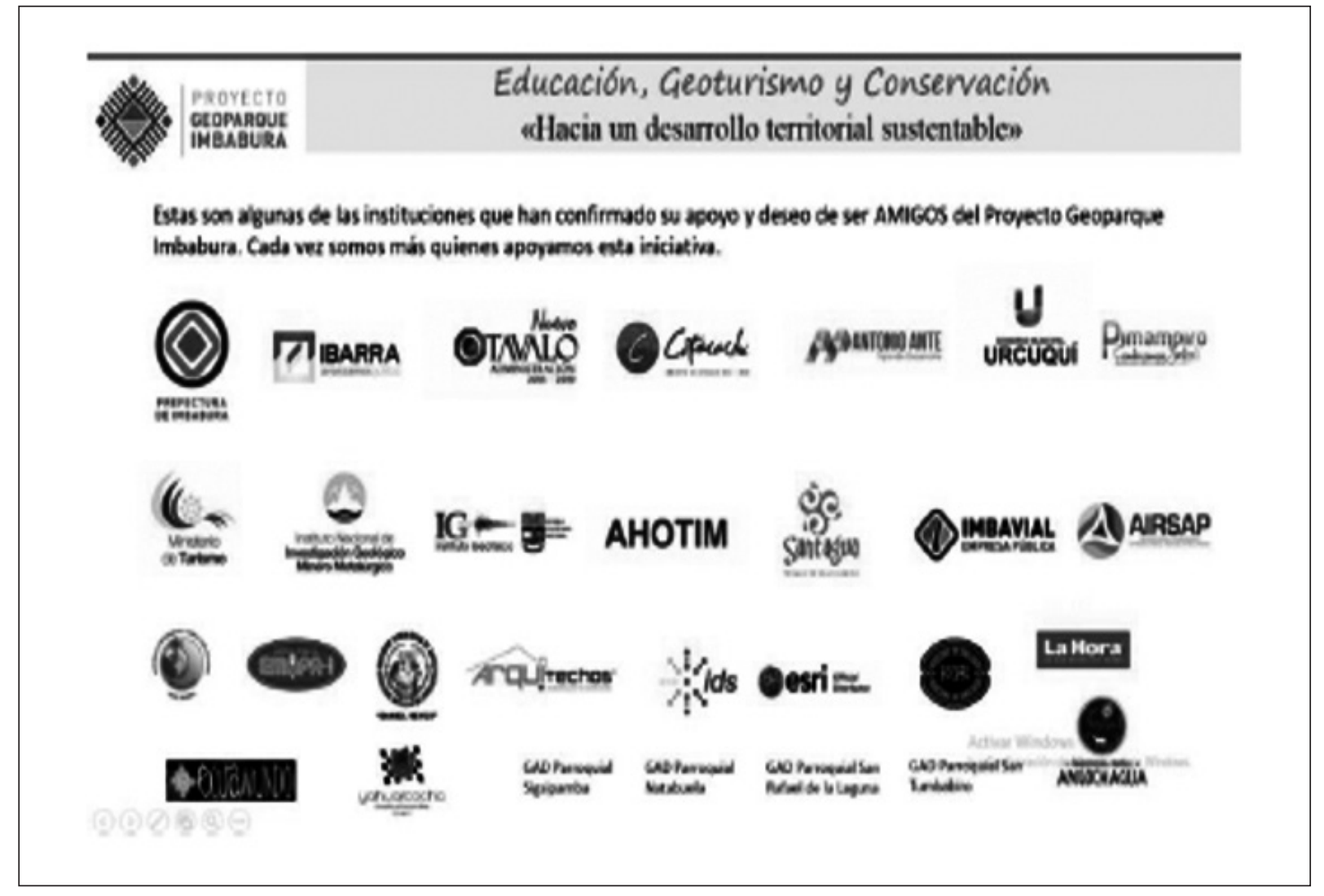

Figura 10. Logotipos de los “Amigos del Geoparque Imbabura” (Comité de Gestión Proyecto Geoparque Imbabura, 2017). 
evidencia de la visión compartida por lograr mejorar la calidad de vida de los habitantes de Imbabura. El contacto con otros aspirantes a lograr el reconocimiento como Geoparques Mundiales, como el Geoparque Volcán Tungurahua y Napo-Sumaco, en Ecuador, es cercana y familiar. Como producto de esta política, las instituciones técnicas-científicas, afines al proyecto, cooperan con sus conocimientos para fundamentar la propuesta y como resultado, poco a poco mediante actividades de socialización y comunicación, la comunidad está mejor informada y se empodera con la iniciativa.

Como política institucional, el Proyecto Geoparque Imbabura, impulsa sus acciones en concordancia con los Objetivos de Desarrollo Sostenible, con los artículos pertinentes a los derechos de la $\mathrm{Pa}$ cha Mama, consagrados en la Constitución del Ecuador y en el Programa de Gobierno de la Prefectura de Imbabura.

Con este propósito, el Comité de Gestión ha sido conformado con los representantes de los acto- res locales, para que exista participación amplia, con aportes y sentido crítico de la realidad y del escenario prospectivo al que se dirige. Así, en el Proyecto Geoparque Imbabura, la función Geoeducación está ubicada en primer lugar, porque es fundamental inculcar en la población a través del conocimiento del entorno, una conducta amigable con el ambiente.

$\mathrm{La}$ articulación de actores locales, públicos y privados, del sector rural y urbano, paso a paso, han generado sinergias. El espíritu emprendedor de la población se evidencia en la apertura de medios de vida individuales y asociativos que demuestran una mejor calidad de vida para toda la comunidad.

En noviembre 30 de 2015, el Comité de Gestión del Proyecto Geoparque Imbabura, realizó una convocatoria pública, como política de "participación ciudadana", para recibir propuestas de logotipos; se recibieron 57 propuestas de las cuales el proyecto ganador es el que se muestra en la figura 11, convirtiéndose en la imagen oficial del Proyecto Geoparque Imbabura.

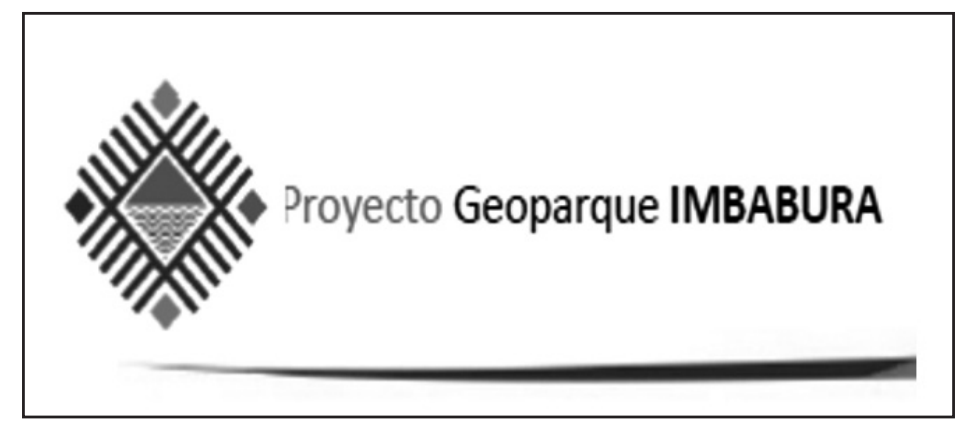

Figura 11. Logotipo ganador del concurso (Comité de Gestión Proyecto Geoparque Imbabura, 2017).

En cumplimiento de una política de participación ciudadana, se puso en ejecución, de forma permanente, la campaña "Amigos" del Proyecto Geoparque Imbabura. Mediante este instrumento, personas naturales, jurídica, públicas y privadas, organizaciones de la sociedad civil, presentaron sus adhesiones al proyecto y se comprometieron a trabajar juntos por el fortalecimiento de la identidad provincial compartiendo la ilusión de alcanzar la denominación "Geoparque Mundial de la UNESCO".

En la provincia, las unidades educativas a nivel medio, las organizaciones de la sociedad civil, la academia, el sector privado, el sector público, se unieron a la propuesta del PGI, y se sumaron con sus propios roles para participar activamente en el proceso.

Se elaboró el dossier en dos versiones: español e inglés, el mismo contiene todos los documentos recopilados y generados desde el año 2015 los mismo que sirvieron para fundamentar científicamente y poner en valor los patrimonios geológico, natural, antropológico, cultural, gastronómico de la provincia de Imbabura. El dossier final se presentó el día 7 de febrero del 2019 a la UNESCO, cumpliendo con todas las formalidades del caso y respetando las fechas exigidas para el mismo.

Este proceso ha significado un resultado de aprendizaje en un tema relativamente nuevo en la provincia, pero que ha logrado que sus actores, desarrollen distintos tipos de habilidades y destrezas que bien podrían servir para el acompañamiento técnico y/o asesoría de otros proyectos afines en el país.

Lo que resta es esperar a la declaratoria de los nuevos geoparques mundiales que realizará la UNESCO el 17 de abril 2019 desde la ciudad de 
París, donde se conocerá si la provincia de Imbabura logra su reconocimiento como GEOPARQUE MUNDIAL DE LA UNESCO, con la alegría, pero

\section{Referencias}

Aguas, C. (2018). Zuleta, segundo destino turístico en Imbabura, según encuesta. Consultado 24 de noviembre, 2018, Obtenido de: https://www. elnorte.ec/ibarra/zuleta-segundo-destino-turistico-en-imbabura-segun-encuesta-XA211088

Asamblea Nacional del Ecuador. Código Orgánico de Organización Territorial (COOTAD) (2010). Quito, Ecuador.

Carcavilla L., Belmonte, A., Durán, J., \& Hilario, A. (2011). Geoturismo: concepto y perspectivas en España, Enseñanza de las Ciencias de la Tierra, 19(1), 81-94.

Carcavilla, L., \& Cortés, Á.G. (2018). Geoparques. Significado y funcionamiento. Madrid: Instituto Geológico y Minero de España

Biblioteca de Investigaciones. (2018). Ciencias de la tierra. Consultado 11 de noviembre, 2018, Obtenido de: https://bibliotecadeinvestigaciones. wordpress.com/ciencias-de-la-tierra/ a la vez la gran responsabilidad que esa denominación implica.

Comité de Gestión Proyecto Geoparque Imbabura. (2017). Dossier Proyecto Geoparque Imbabura 2017. Ecuador: Comité de Gestión Proyecto Geoparque Imbabura. (2017). 54p.

SEMOP-MAE (2017). Socio Bosque SEMOP. Obtenido de: http://semop.ambiente.gob.ec:8085/ Sisbweb/

Denzin, N. (1989). Interpretative Interactionism. Londres: Sage

EcuRed. (2018). Ciencia de la tierra. Consultado 11 de november, 2018, Obtenido de: https://www. ecured.cu/Ciencia_de_la_tierra

UNESCO. (2018). Programa Internacional de Geoparques de la UNESCO. Obtenido de: http:// www.unesco.org/new/es/office-in-montevideo/natural-sciences/international-programme-for-geosciences-and-geoparks/unesco-global-geoparks 